\title{
A Reputation Management Framework Based on Global Trust Model for P2P Systems*
}

\author{
Jingtao Li, Xueping Wang, Yongqiang Chen, and Gendu Zhang \\ School of Information Science \& Engineering, Fudan University, Shanghai, 200433, China
}

\begin{abstract}
A framework based on global trust model, called SRGTrust, is proposed for reputation management in P2P systems. SRGTrust assigns each peer a unique global trust value, which reflects the rating that the system as a whole gives to a peer. SRGTrust does not need any pre-trusted peers to ensure the convergence of the algorithm and invalidates one of the basic assumptions used in the previous models. Experiments show that SRGTrust converges quickly and significantly decreases the number of inauthentic files in the system.
\end{abstract}

\section{Introduction}

Motivation. In decentralized peer-to-peer (P2P) file-sharing systems, due to the anonymous and self-organization nature of peers, they have to manage the risk involved with the transactions without prior experience and knowledge about each other's reputation. One way to address this uncertainty problem is to develop strategies for establishing reputation systems [2,3] that can assist peers in assessing the level of trust they should place on the quality of resources they are receiving.

Challenge. The very core of the reputation mechanism in a $\mathrm{P} 2 \mathrm{P}$ system is to build a distributed reputation management system that is efficient, scalable, and secure in both trust computation and trust data storage and dissemination. The main challenge of building such a reputation mechanism is how to effectively cope with various malicious collectives of peers who know one another and attempt to collectively subvert the system by providing fake or misleading ratings about other peers $[2,3]$.

Contribution. We present a framework, called SRGTrust (Global Trust model based on Similarity-weighted Recommendations), for reputation management in P2P systems, which includes a global trust model for quantifying the trustworthiness (section 2), and a decentralized implementation of such model over a structured P2P overlay network (section 3). Previous global trust models such as eigentrust [3] are based on the assumption that the peers with high trust value will give the honest recommendation. We argue that this assumption may not hold in all cases. In SRGTrust, each peer $i$ is assigned a unique global trust value that based on similarity-weighted recommendations of the peers in the network who has interacted with peer $i$; Finally, A series of simulation-based experiments show that the SRGTrust algorithm is robust and efficient to cope with various malicious collectives (section 4).

This work is supported by the National Natural Science Foundation of China (No. 60373021). 


\section{The Trust Model}

Assume $n$ denotes the number of the peers in a system. Each peer $i$ rates another peer $j$ from which he tries to download files by rating each download as either positive or negative, depending on whether $i$ was able to download an authentic file from $j$ or not. The sum of the ratings of all of $i$ 's interactions with $j$ is called a local trust value $S_{i j}$. $S_{i j}=G_{i j}-F_{i j}$ wherein $G_{i j}$ denotes the number of positive downloads and $F_{i j}$ denotes the number of negative downloads.

A normalized local trust value $\boldsymbol{L}_{i j}$ is defined as follows:

$$
L_{i j}=\frac{\max \left(S_{i j}, 0\right)}{\sum_{j} \max \left(S_{i j}, 0\right)} .
$$

$L_{i j}$ is a real value between 0 and 1 , and $\sum_{j} L_{i j}=1$. If $\sum_{j} \max \left(S_{i j}, 0\right)=0$, let $L_{i j}=T_{i} / n . L_{i i}=0$ for every peer $i$; otherwise, $i$ can assigns arbitrarily high local trust values to itself.

One critical step in our model is to compute the similarity between rating opinions of peers and then to weight the peers' recommendations by that value. The similarity between them is measured by computing the cosine of the angle between these two vectors [1]. The similarity between peers $i$ and $j$, denoted by $\boldsymbol{C}_{i j}$, is given by

$$
C_{i j}=\frac{B_{i} * B_{j}}{\left\|B_{i}\right\| \cdot\left\|B_{j}\right\|}
$$

where $" *$ " denotes the dot-product of the two vectors. Here $\boldsymbol{B}_{i}$ denotes the rating opinion vector of peer $i$, defined as $B_{i}=\left[B_{i i}, B_{i 2}, \ldots, B_{i n}\right]$ where $B_{i k}(k=1, \ldots, n)$ is the rating opinion of peer $i$ on peer $k . B_{i k}$ is defined as follows:

If $i \neq k$, and $G_{i k}+F_{i k}=0$, then $B_{i k}=0$;

if $i \neq k$, and $G_{i k}+F_{i k}>0$, then $B_{i k}=\left\{\begin{array}{ll}G_{i k} /\left(G_{i k}+F_{i k}\right) & G_{i k} \geq F_{i k} \\ -F_{i k} /\left(G_{i k}+F_{i k}\right) & G_{i k}<F_{i k}\end{array}\right.$.

For each peer $i$, we let $B_{i i}=1+\varepsilon$ where $\varepsilon$ is an arbitrarily small positive constant.

A global trust value vector, $\boldsymbol{T}=\left[T_{1}, T_{2}, \ldots, T_{n}\right]^{T}$, is given by

$$
T_{i}=\sum_{k \in U_{i}}\left(L_{k i} \cdot C_{k i} \cdot T_{k}\right)
$$

where $T_{i}$ is the global trust value of peer $i . U_{i}$ denotes the set of the peers who have interacted with peer $i$. The global trust value of peer $i$ is the sum of the weighted recommendations of the peers that have interacted with $i$ in a single iteration. After several iterations of asking friends of friends by using the above formula, peer $i$ will have a view of the entire network. The credibility of the recommendations of a peer is different from that of the peer itself. So, different from eigentrust [3], we weight the recommendations of peer $k$ by the similarity between peers $k$ and $i$. 


\section{Distributed Implementation}

Trust Data Management. There is no central database in P2P environments. Trust data that are needed to compute the trust measure for peers are stored across the network in a distributed manner. Each peer has a trust value manager that is responsible for feedback submission and trust evaluation, a small database that stores a portion of the global trust data, and a data locator for placement and location of trust data. In our implementation, the DHT algorithm, Chord [4], is used to assign trust value managers to peers. The detailed methods will not be given here due to space constraint.

The SRGTrust Algorithm is executed to compute a global trust value vector in a dynamic and decentralized fashion at each peer.

Primitives. Submit $\left(I D_{i},\left(I D_{j}, I D_{k}\right)\right.$, Value1, Value2): Submitting Valueland Value2 to the trust value managers of peer $i$. We use the Value1 and Value2 to refer to the normalized local trust value that peer $j$ place in $k$ and rating opinion vector of peer $j$, the meaning will be clear from context; Query $\left(I D_{j}, T_{j}, L_{j j}, B_{j}\right)$ : Querying the global trust value, recommendation to peer $i$, and rating opinion vector of peer $j$.

Algorithm 1. SRGAgorithm1, peer $i$ as an ordinary peer.

Update_SubmitTrustdata( ) // submits its rating $\left(G_{i j}, F_{i j}\right)$ to peer $j$ after a transaction

$\left\{\quad\right.$ If (a good transaction ) $G_{i j} \leftarrow G_{i j}+1$;

else $\quad F_{i j} \leftarrow F_{i j}+1$;

$\left.\operatorname{Submit}\left(I D_{i},\left(I D_{i}, I D_{j}\right), G_{i j}, F_{i j}\right) ; \quad\right\}$

// submits $G_{i j}$ and $F_{i j}$ to $M_{i}$, and triggers the UpdateLocaltrust( ) in $M_{i}$

Algorithm 2. SRGAgorithm2, peer $i$ as a trust value manager of peer $u$.

UpdateLocaltrust( ) // updates $L_{u v}$ and $B_{u v}$ after receiving a Submit primitive from $u$

$\left\{\right.$ Verify the consistency of $G_{u v}$ and $F_{u v}$;

Compute $S_{u v}, L_{u v}, B_{u v}$;

Submit $\left.\left(I D_{v},\left(I D_{w}, I D_{v}\right), L_{u v}, n u l l\right) ; \quad\right\} \quad / /$ submits $L_{u v}$ to $M_{v}$

CalcGlobaltrust( )

// computes the global trust value of peer $u$

for ( every $j \in U_{u}(j \neq u)$ )

$\left\{\quad\right.$ Query $\left(I D_{j}, T_{j}, L_{j u}, B_{j}\right)$;

$C_{j u} \leftarrow \frac{B_{j} * B_{u}}{\left\|B_{j}\right\| \cdot\left\|B_{u}\right\|} ; \quad \quad / /$ computes the similarity between peers $u$ and $j$

$\left.T_{u} \leftarrow T_{u}+L_{\mathrm{ju}} \cdot C_{\mathrm{ju}} \cdot T_{\mathrm{j}} ; \quad\right\}$

return $\left.T_{\mathrm{u}} ; \quad\right\}$

\section{Experiments}

Simulation Setup. As a test bed for the experiments, we use the Query Cycle Simulator [5] which simulates a P2P file-sharing network. Trust values in this network are used to bias download sources. We shall demonstrate the algorithms' performance under two typical threat models. Threat Model IM: Individual malicious peers, called IM peers, always provide an inauthentic file when selected as a download source and they always set their satisfaction values to $S_{i j}=F_{i j}-G_{i j}$; Threat Model CM: 
Malicious peers, called CM peers, always provide an inauthentic file when selected as a download source and each CM peer $i$ let $L_{i j}=1 /\|C M s\|$ for any $j \in \mathrm{CMs}$ where CMs denotes the set of all CM peers, and $\|\mathrm{CM}\|$ denotes the total of all CM peers.

Results. Proportion of Authentic Downloads (PAD), the ratio of the number of authentic downloads to the number of all downloads viewed by all good peers when we use three algorithms separately, are showed in Fig. 1. The PAD is higher than $80 \%$ even if IM, or CM peers make up a half of all peers in the network when our scheme is activated.

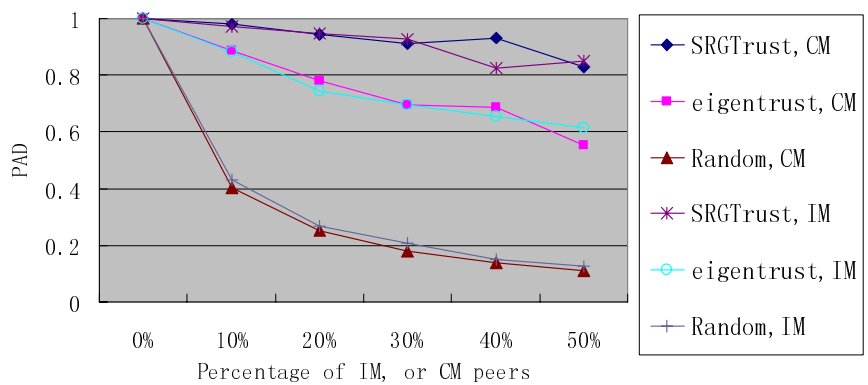

Fig. 1. PAD against different percentage of malicious peers. "Random" means a P2P system where no reputation system is implemented. We set up a network consisting of 500 peers. The initial distribution of the global trust values is a uniform probability distribution over all $n$ peers, that is $\mathrm{T}_{i}=1 / n(\mathrm{i}=1, \ldots, n)$. After issuing a query, a peer waits for incoming responses from the peers that have the file he is looking for, selects a download source whose global trust value is the highest among those peers, and downloads the file. The last two steps are repeated until the peer receives a complete, authentic copy of the file.

\section{References}

1. Sarwar, B., Karypis, G., Konstan, J., Riedl, J.: Item-Based collaborative filtering recommendation algorithms. Proceedings of the 10th International World Wide Web Conference. ACM Press. (2001) 285-295

2. Xiong, L., Liu, L.: PeerTrust: Supporting Reputation-Based Trust for Peer-to-Peer Electronic Communities. IEEE TRANSACTIONS ON KNOWLEDGE AND DATA ENGINEERING 16(7). IEEE Press. 843-857

3. Kamvar, S.D., Schlosser, M.T.: The eigentrust algorithm for reputation management in P2P networks. Proceedings of the 12th international conference on World Wide Web. ACM Press. (2003) 640-651

4. Stoica, I., Morris, R., Karger, D.: Chord: A scalable peer-to-peer lookup service for Internet applications. Technical Report. MIT. (2002). http://pdos.csail.mit. edu/chord/

5. Schlosser, M.T., Condie, T.E., Kamvar, S.D.: Simulating a File-Sharing P2P Network. Proceedings of First Workshop on Semantics in P2P and Grid Computing. (2003) 69-80 